\title{
MicroCT-Based Phenomics in the Zebrafish Skeleton Reveals Virtues of Deep Phenotyping in a Distributed Organ System
}

Matthew Hur, ${ }^{1}$ Charlotte A. Gistelinck, Philippe Huber, Jane Lee, Marjorie H. Thompson, Adrian T. Monstad-Rios, Claire J. Watson,

Sarah K. McMenamin, ${ }^{3}$ Andy Willaert, ${ }^{2}$ David M. Parichy,

Paul Coucke, and Ronald Y. Kwon ${ }^{1}$

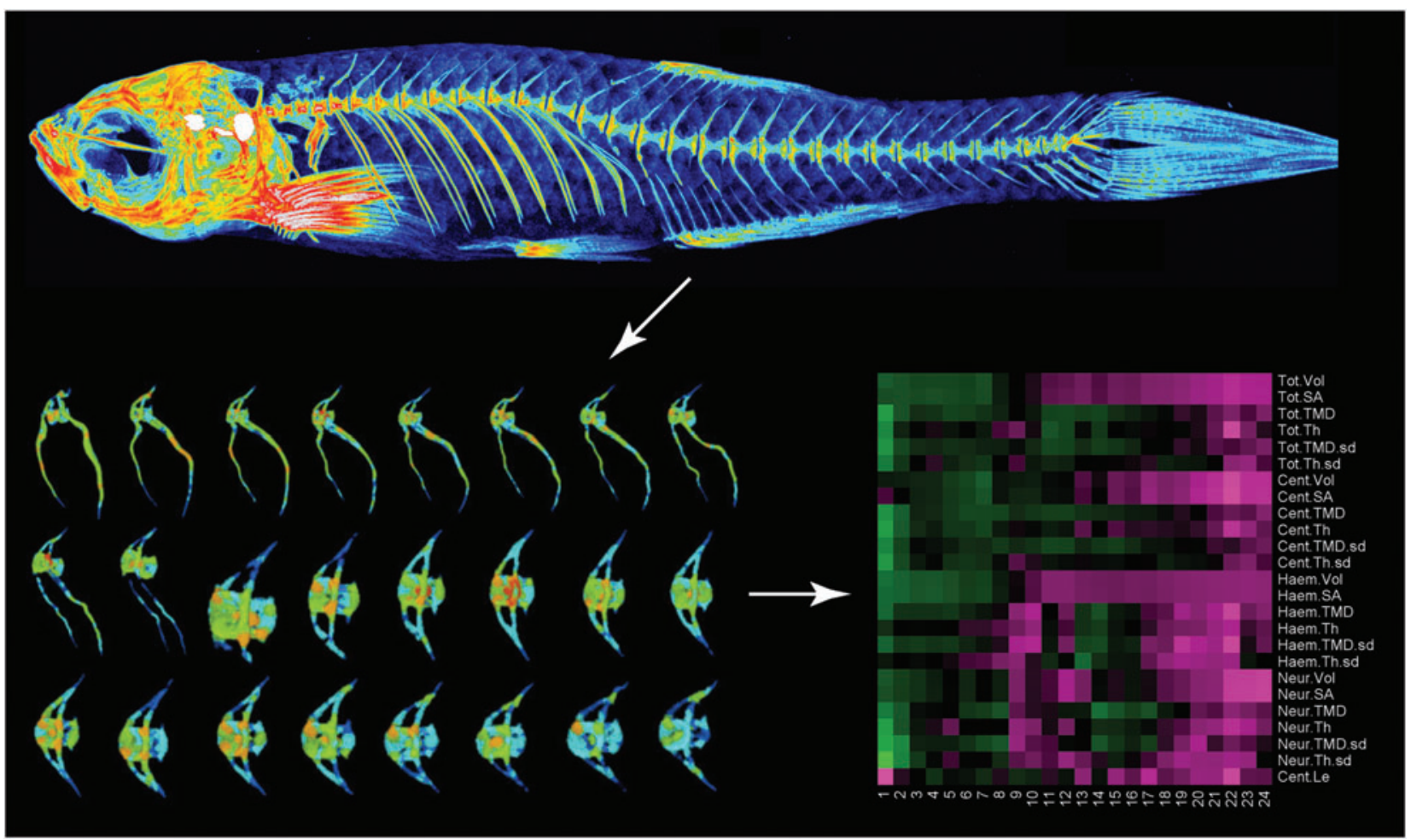

FIG. 1. Using whole-body microCT scans (top), FishCuT isolates individual vertebrae (bottom left), segments each vertebra into three elements, and computes traits in each element. Standard scores are computed and arranged into "skeletal barcodes" that facilitate data visualization (bottom right). Within the barcode, each column represents a single vertebra and each row represents a phenotypic measure. Color images available online at www.liebertpub.com/zeb

${ }^{1}$ Department of Orthopaedics and Sports Medicine, University of Washington, Seattle, Washington.

${ }^{2}$ Center for Medical Genetics, Ghent University, Ghent, Belgium.

${ }^{3}$ Biology Department, Boston College, Chestnut Hill, Massachusetts.

${ }^{4}$ Department of Biology, University of Virginia, Charlottesville, Virginia. 
Keywords: phenomics, zebrafish, vertebra, skeleton, bone, mineralization

$\mathbf{T}$

He ABILITy to CHARACTERIZE PHENOMES- that is, to acquire in-depth phenotypic profiles at the scale of the whole organism-holds promise to enhance our understanding of genetic variation. Yet, phenomic profiling in vertebrates remains limited. Hur et al. ${ }^{1}$ developed microCT-based methods and a segmentation algorithm, FishCuT, enabling profiling of hundreds of phenotypic measures at a large number of anatomical sites in the axial skeleton of adult zebrafish (Fig. 1). Approximately 30,000 data points and $\sim 3500$ skeletal elements were analyzed. Vertebral patterns conferred heightened sensitivity, with similar specificity, in discriminating mutant populations compared with analyzing individual vertebrae in isolation. Skeletal phenotypes associated with human brittle bone disease and thyroid stimulating hormone receptor hyperactivity were identified. Finally, allometric modeling-based methods were developed to aid in the discrimination of mutant phenotypes masked by altered growth. This study establishes virtues of microCT-based phenomics in zebrafish. FishCuT is available for download as a beta release. ${ }^{2}$

\section{References}

1. Hur M et al. MicroCT-based phenomics in the zebrafish skeleton reveals virtues of deep phenotyping in a distributed organ system. Elife 2017;6:e26014.

2. Kwon RY. FishCuT. Github. 2860a3a. 2017. Available at https://github.com/ ronaldkwon/FishCuT

Address correspondence to:

Ronald Y. Kwon, PhD

Department of Orthopaedics and Sports Medicine

University of Washington

325 Ninth Avenue, Box 359798

Seattle, WA 98104

E-mail: ronkwon@uw.edu 